\title{
Lessons Learned from the Japan Obstetric Compensation System for Cerebral Palsy: A Novel System of Data Aggregation, Investigation, Amelioration, and No-Fault Compensation
}

\author{
Shin Ushiro, Antonio Ragusa, \\ and Riccardo Tartaglia
}

Cerebral palsy is a pathological condition whose prevention and treatment have been immensely studied by experts in perinatal medicine and pediatric neurology. Despite this, it is still one of the main concerns nowadays in many countries, not only for scientific reasons but for legal ones also. For instance, in Japan, an increase in lawsuits relating to cerebral palsy was observed more than a decade ago, after healthcare resources in perinatal medicine had increasingly shrunk and had become fragile under the growing burden for physicians and midwives to provide advanced treatment, emergent care, high-risk treatment, and so on. Young physicians did not specialize in obstetrics because of the increased burden, which gave rise to a vicious cycle of shrinking resources in perinatal medi-

\section{S. Ushiro}

Division of Patient Safety, Kyushu University Hospital, Fukuoka, Japan

Japan Council for Quality Health Care, Tokyo, Japan e-mail: ushiro@surg2.med.kyushu-u.ac.jp

A. Ragusa $(\bowtie)$

Obstetrics and Gynecology Department,

Fatebebefratelli Hospital; Isola Tiberina, Rome, Italy

R. Tartaglia

Institute of Management, School of Advance Studies

Sant'Anna, Pisa, Italy cine. To address this issue, the Japan Obstetric Compensation System for Cerebral Palsy (JOCS-CP) was urgently introduced in 2009 to investigate, develop preventive measures, and award monetary compensation on a no-fault basis, with the Japan Council for Quality Health Care (JQ) as its operating organization (Fig. 33.1). It has so far produced annual reports on the prevention of cerebral palsy for nine consecutive years including numerical data and specific themes relating to the occurrence and prevention of cerebral palsy. The success of the system is a good reference for responding to adverse events which may happen in and have a vast impact on perinatal care. Therefore, this chapter focuses on cerebral palsy with primary reference to materials published by the JOCS-CP in the field of perinatal medicine. The aim of this chapter is to learn about the issues mentioned above and to discuss the significance and impact of introducing a nationwide system like the JOCS-CP. It describes knowledge and idea to questions of "Why cerebral palsy is highlighted among adverse event in obstetrics?", "How the no-fault compensation/investigation/prevention system could be introduced?", "What has been achieved by the system?", and "How cerebral palsy is prevented?". 


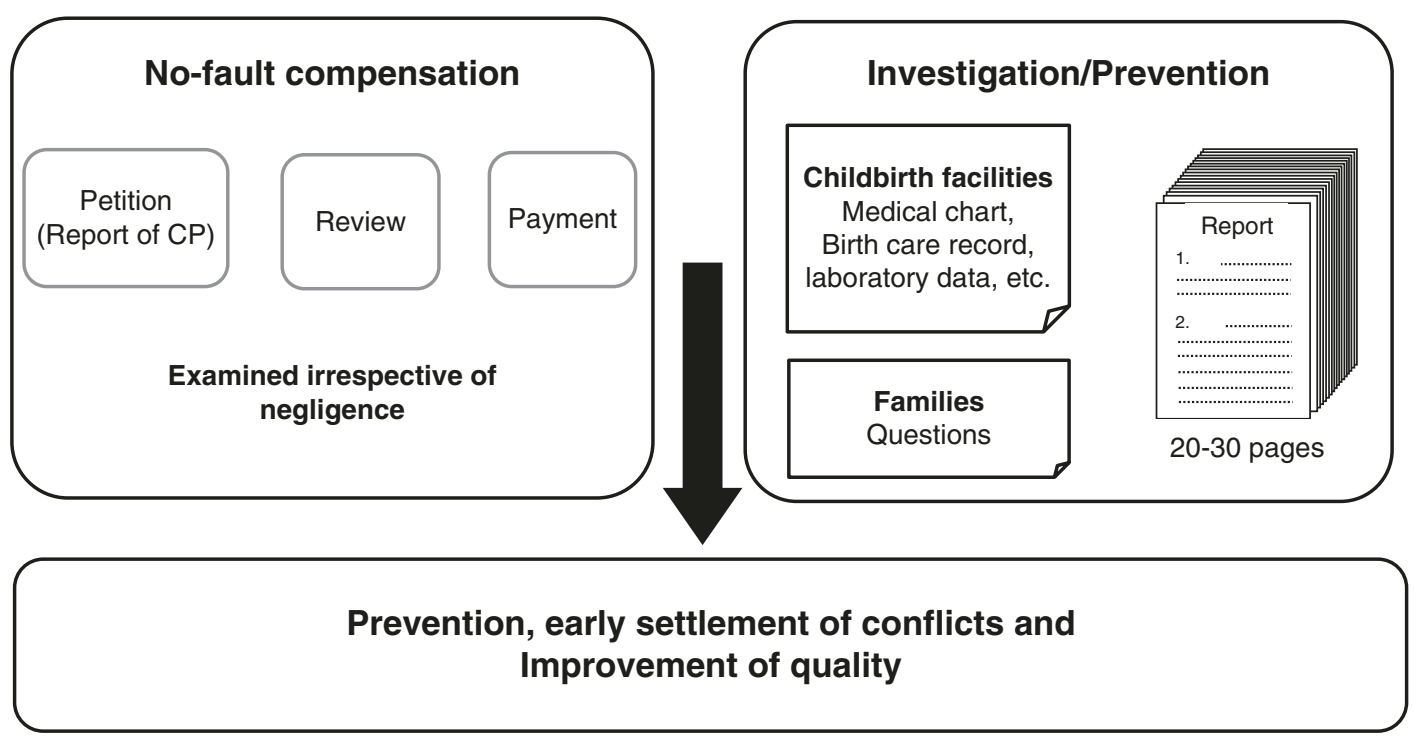

Fig. 33.1 No-fault compensation/investigation/prevention system for cerebral palsy. (The Japan Obstetric Compensation System (2009))

\subsection{Context for the Introduction of the JOCS-CP: Increasing Conflict Over Cerebral Palsy and Hopes for a No-Fault Compensation System}

According to the numbers of lawsuits filed in 2004 against each medical specialty, per registered physician, the obstetrics and gynecology rate was the highest (12.4\%), a heavy burden for all obstetricians involved. One assumption made about the obstetrics lawsuits was that a significant portion of them related to cerebral palsy. It should be noted that recent studies have speculated that most cases of cerebral palsy were not related to procedures performed by obstetricians and midwives, although there actually are a small number of cases caused by violations of current quality-of-care guidelines and/or standards. In general, it is difficult to distinguish between negligence and other causes of cerebral palsy. For example, a child may develop profound cerebral palsy after a normal or seemingly normal pregnancy and delivery. The onset of cerebral palsy is likely to provoke turmoil leading to legal action taken by the family, which raises questions about the delivery procedure itself and the family's worries and anxieties about the long-term care needed for rearing their disabled child. To address this issue, obstetricians and relevant experts in Japan have long studied and explored the possibility of introducing a no-fault compensation system for cerebral palsy at the national scale to reverse the persistent high frequency of conflict around cerebral palsy. Concurrently, the Japan Medical Association (JMA) has been considering no-fault compensation since the early 1970s. Its 1972 report entitled "The Legal Proceedings for Medical Accidents and the Relevant Theory" touched upon the following three items regarding institutionalization of such a system:

1. Prompt compensation should be provided in cases of medical accidents for which a physician is deemed responsible after a thorough review of the case.

2. A unique national compensation scheme should be devised to cover cases of accidents 
not attributed to a medical procedure conducted by a physician, that is, cases that were inevitable during clinical care.

3. A unique national system should be established for settling disputes, functioning independently of the judicial court system.

Regarding the first point, the JMA Physician's Liability Compensation System was launched in 1973 in line with the indemnity insurance system. However, neither the second nor the third point were realized in the following decades. In the early 2000s, concerns about patient safety, the shortage of obstetricians and pediatricians, and low national birth rates rapidly emerged in Japan. This eventually prompted the JMA to publish a report entitled "Toward Compensation in Cases Related to Medical Management and Procedures" in January of 2006. It states that "although it is ideal to launch a compensation system to cover entire medical specialties, a compensation system with a narrower scope for cases with neurological sequela, i.e., cerebral palsy, should be given the highest priority for institutionalization." The JMA board member who supervised the compilation of the report was an obstetrician and vice president who, years later, became the president of the Japan Association of Obstetricians and Gynaecologists (JAOG). In August of 2006, the JMA published another report compiled by a project committee on the progress of the implementation of a compensation system for disabilities caused by cerebral palsy. It set forth concrete and detailed reasons to launch a no-fault compensation system specific to cerebral palsy. The project was further promoted by a report from the Policy Research Committee of the Liberal Democratic Party (LDP) which was at the time and still is a member of a leading political alliance. The report was titled "The Framework of No-fault Compensation System for Obstetric Care," commonly referred to as the "LDP Framework." The report described the perceived significance of launching no-fault compensation system for cerebral palsy by declaring, "It usu- ally is difficult to identify malpractice regarding an adverse event during delivery, and those cases are apt to be contested in court. The frequency of disputes is one reason for the shortage of obstetricians." The LDP Framework proposes that, to secure safe and trustworthy perinatal care that benefits obstetricians, patients, and families, a new system should: (1) compensate patients who develop impairments possibly caused by adverse obstetric events, (2) swiftly resolve and settle all disputes, and (3) improve the quality of obstetrical care by investigating cerebral palsy cases. Immediately upon the release of the LDP Framework, significant official and unofficial attention focused on JQ, proposing that it should be an operating organization for the novel system. JQ was the natural choice because it had previously conducted projects on quality and safety improvement, such as hospital accreditation and Adverse Event Reporting and Learning Systems. In response to high expectations, JQ established in 2007 the Division of Introductory Work on the Japan Obstetric Compensation System for Cerebral Palsy (JOCS-CP) as a temporary agent to introduce the JOCS-CP in Japanese healthcare system. At this point, it should be noted that political decision added momentum to the growing demand by academic society to launch the JOCS-CP; JQ was considered to be the right choice to operate the JOCS-CP because it was a neutral body related to quality and safety improvement in healthcare. The secretary installed in JQ for working on the JOCS-CP during preparatory period intensively studied potential obstacles for the launch of a system and, in January of 2008, finally issued a report which described the detailed design of the system. After careful review of the report, the JQ board accepted the role of managing organization of the system in March of 2008. Subsequently, the JOCS-CP was launched on the 1st of January 2009, and has been in operation since, undergoing a revision in 2015 that saw the expansion of eligibility criteria with the full support of stakeholders. 


\subsection{The Meaning of "No-Fault Compensation" in the JOCS-CP}

The term "no-fault compensation" could be defined in multiple ways. Besides the JOCS-CP, another compensation system in Japan-a system regarding medication side effects, managed by the governmental Pharmaceuticals and Medical Devices Agency (PMDA) - has been described as a no-fault compensation system because it compensates individuals who suffer medication side effects provided that the medication was prescribed and administered appropriately and that the medication is the probable cause of the adverse side effects. The system is designed based on the idea that adverse side effects can occur even when appropriate medication is prescribed and administered. On the other hand, no-fault compensation might refer to a system which provides compensation no matter the degree of any suspected negligence. This is an idea of the JOCS-CP which is different from the one adopted by the civil judicial system.
Specifically, the LDP Framework states that the JOCS-CP will compensate patients with cerebral palsy that was possibly caused by delivery procedures regardless of the extent of obstetrical negligence. Therefore, cerebral palsy cases obviously caused by congenital defects, such as cerebral anomalies, and thus not related to the obstetrical procedures are not eligible for compensation under the system.

\subsection{Compensation Driven by the Indemnity Insurance Mechanism}

The two main pillars of the JOCS-CP are compensation and investigation/prevention (Fig. 33.1). Compensation works through a mechanism of indemnity insurance services provided by the alliance of indemnity insurance companies under contract with JQ, as indicated in "The Framework of the No-fault Compensation System in Obstetric Care" (Fig. 33.2). As described within this framework, a childbirth

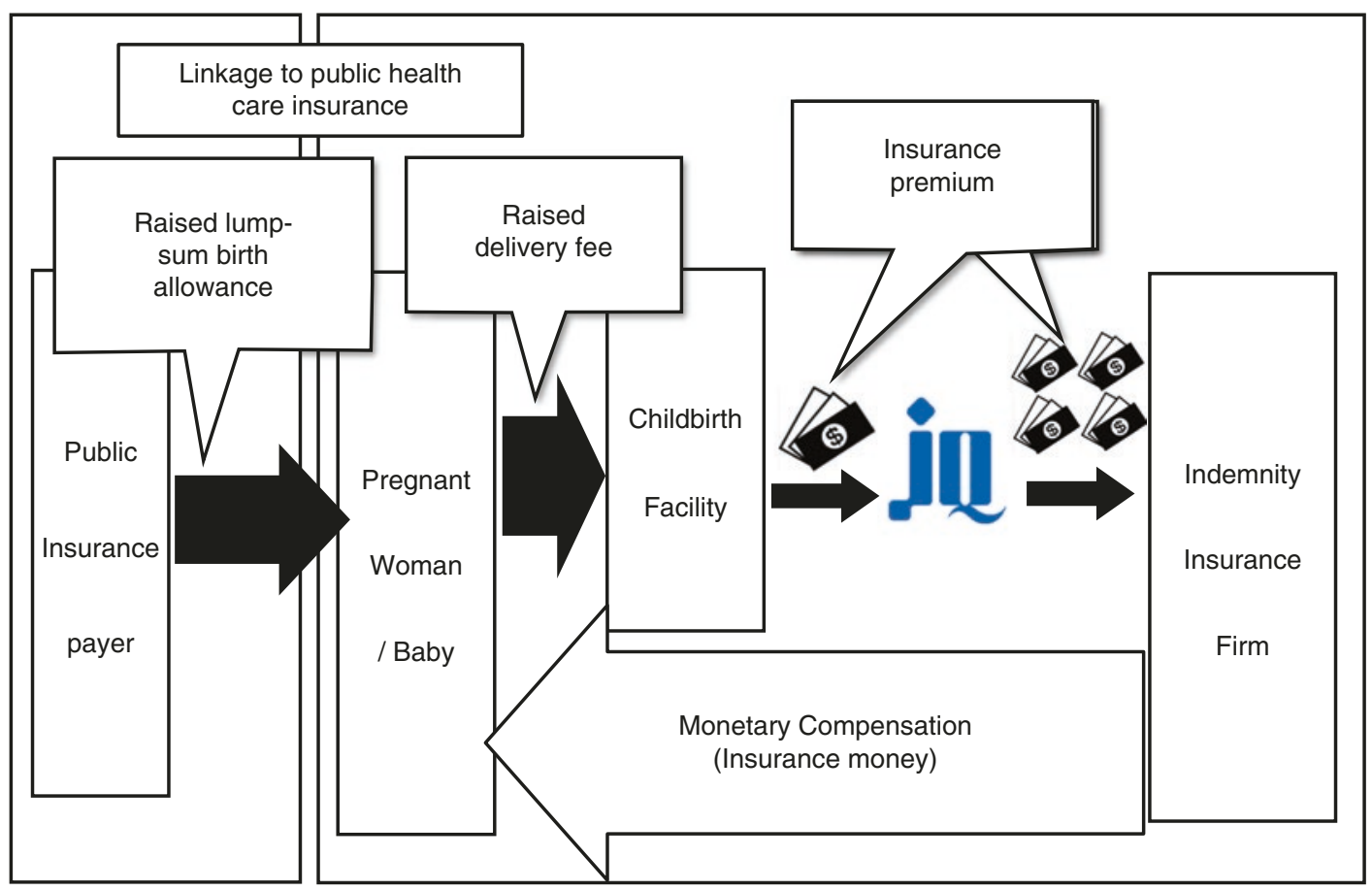

Fig. 33.2 Finance 
facility (1) registers with the JOCS-CP under a unified agreement, and (2) agrees to pay a compensation premium to an indemnity insurance company through JQ when a baby is born and cooperate with JQ for investigation when a baby develops profound cerebral palsy. The facility must notify pregnant women of the compensation for a baby with profound cerebral palsy by submitting the "Registration Certificate for Pregnant Women" to them. Childbirth facilities pay insurance premiums as high as JPY 16,000 (USD 140/EUR 120) which are paid to the indemnity insurance companies through JQ. At the inception of the system, the premium was JPY 30,000 (USD 265/EUR 225) for every baby born between 2009 and 2014. The reason for the subsequent reduction of the premium will be described later. JQ mediates the transaction, collecting payments from the registered childbirth facilities and regularly depositing lump sums with the indemnity insurance companies. Children who develop profound cerebral palsy are assessed by the Review Committee to see whether they meet the eligibility criteria for compensation. When a case is approved, monetary compensation is awarded as required under the compensation agreement between the childbirth facility and the family. For practical reasons, the compensation is directly paid to the patient's guardian by the indemnity insurance company. According to LDP Framework, the Ministry of Health, Labour and Welfare (MHLW) increased the lump sum childbirth compensation by an amount equivalent to that of the insurance premium in response to the likely increase in childbirth costs set by childbirth facilities. Between 2009 and 2014, the amount was JPY 30,000 (USD 265/EUR 225), and, since 2015, the amount has been JPY 16,000 (USD 140/EUR 120). Thus, the lump sum payment of public health insurance for childbirth has served as a financial source of compensation in the JOCS-CP. This link between compensation and public health insurance appears to qualify the JOCS-CP as a quasi-public system. The amount of lump sum compensation was stable after the system launched, at JPY 420,000 (USD 3700/ EUR 3150) for babies born in registered childbirth facilities; then, in 2015, the insurance premiums reduced to JPY 16,000 from JPY 30,000. The standard compensation agreement stipulates that lump sum payments should generally be paid within 60 days of a petitioner's complete submission, while it has actually been paid as swift as 25 days or less after the petition. Similarly, annual installment should be paid within 60 days of the day of the month in which the child was born or of the receipt of all required forms, whichever comes first.

\subsection{Monetary Compensation}

The total monetary compensation per patient amounts to JPY 30,000,000 (USD 266,000/EUR $225,000)$ in two forms of payment: a lump sum of JPY 6,000,000 (USD 53,300/EUR 45,200) and annual installments of JPY 24,000,000 (USD 212,700/EUR 179,800). Installments of JPY 1,200,000 (USD 18,600/EUR 15,800) are paid once a year for 20 years starting in the birth year of the claimant and ending on their 19th birthday. For example, regarding the petition and review process of events that occurred in the first year of a baby's life, the review of all cases for children born in 2009 must be completed by 2015 because a petition may be filed until the claimant's fifth birthday (as stated in the standard compensation agreement). Petitions for patients born in 2010 (open until 2016) or later are processed under the same guideline.

\subsection{Epidemiology of Adverse Events}

\section{Prevalence of cerebral palsy}

The prevalence of cerebral palsy has been reported on by various research groups. In designing the JOCS-CP, JQ explored existing data on Japanese childbirth from Okinawa Prefecture, Himeji City, and a part of the Tokyo metropolitan area. Among these studies, data from Okinawa Prefecture was statistically accurate enough to be used in the designing process of the JOCS-CP. It gave a 
figure of 2.3 cases of cerebral palsy per 1000 live births which is comparable to rates determined in other countries.

2. Estimates of annual numbers of eligible individuals in the JOCS-CP

Eligibility criteria and estimates of the numbers of eligible individuals are crucial elements of the system for appreciating the financial scale. These items were carefully assessed based on cerebral palsy registration data from Okinawa Prefecture, the city of Himeji, and a part of the Tokyo metropolitan area. The approximate number of eligible individuals per year was estimated to be "500-800 persons annually at maximum," which the system took into account to ensure its financial sustainability.

3. Revision of the JOCS-CP including reestimation of the number of eligible individuals

In 2014, it became urgent to improve JOCS-CP financing because the excessive collection of insurance premiums since the system began was raising serious questions in the Japanese parliament, among public payers, in the MHLW, and among relevant entities and clinicians. Although the enumeration of eligible babies born in the first year had not yet been completed, the estimation was made again in 2015 in a more accurate way than that adopted on small scale at the inception of the system. In particular, cerebral palsy registration research had been expanded in Okinawa Prefecture and other institutional data were obtained from consulting facilities for physically impaired persons and relevant medical institutions in Tochigi Prefecture and Mie Prefecture with the help of researchers in those regions. Ultimately, it turned out to be difficult to make use of data from Tochigi Prefecture and Mie Prefecture as they were not reliable enough for making accurate estimations, and so only the data from Okinawa Prefecture was employed to compute a point estimate of 481 and an interval estimate of 340-623, with a 95\% confidence interval. Concurrently, the JOCS-CP's eligibility crite- ria were revised as "born at 32 or more weeks of gestational age and 1400-g or more birth weight" giving rise to new point and interval estimates of 571 and 423-719, respectively. The revised criteria were put into effect in January of 2015.

4. Statistics of eligible individualsEpidemiology of profound cerebral palsy in the JOCS-CP

The review procedure is carried out along with the standard compensation agreement. Briefly, a childbirth facility insured by the JOCS-CP files a petition with JQ, which must be accompanied by certification verifying the diagnosis of profound cerebral palsy by a qualified physician. The certification form must provide relevant information, such as detailed diagnostic and clinical data on the patient. Since scientific knowledge and expertise are necessary for diagnosis, the certification must be issued by physicians certified by the Japanese Society of Child Neurology and/ or registered physicians as defined by Article 15 of the Physically Disabled Persons Welfare Act. Qualified physicians that agree to participate in the review process are voluntarily registered with the JOCS-CP, and their identities affiliations are disclosed on its website; as of June 2019, 511 qualified physicians were registered. The petition undergoes preliminary review and then enters a queue awaiting approval by the Review Committee based on the eligibility criteria. The review process is fundamentally based on documents that demonstrate compatibility with general criteria and exclusion criteria. Upon approval, the JQ board must authorize the petition, which is the final step of the review process. The petitioner (i.e., the childbirth facility) is notified of the approval, which is promptly followed by payment of monetary compensation in the form of a lump sum and annual installments. Petitioners whose cases do not satisfy the general criteria may still be approved in a case-by-case review under the relevant criteria. About 75\% (2755) of the petitions had been approved as of June 2019. Regarding the 
Table 33.1 Statistics of eligible case by birth year (as of June 30, 2019)

\begin{tabular}{l|l|l|l|l|l|l|l}
\hline \multirow{2}{*}{ Birth year } & No. case reviewed & Eligible & \multicolumn{2}{l|}{ Not-eligible } & \multicolumn{2}{l|}{} \\
\cline { 3 - 7 } & & Eligible & $\begin{array}{l}\text { Not } \\
\text { eligible }\end{array}$ & $\begin{array}{l}\text { Preliminary } \\
\text { to review }\end{array}$ & Total & In process & Petition \\
\hline 2009 & 561 & $\mathbf{4 1 9}$ & 142 & 0 & 142 & 0 & Expired \\
\hline 2010 & 523 & $\mathbf{3 8 2}$ & 141 & 0 & 141 & 0 & Expired \\
\hline 2011 & 502 & $\mathbf{3 5 5}$ & 147 & 0 & 147 & 0 & Expired \\
\hline 2012 & 516 & $\mathbf{3 6 1}$ & 155 & 0 & 155 & 0 & Expired \\
\hline 2013 & 476 & $\mathbf{3 5 1}$ & 125 & 0 & 125 & 0 & Expired \\
\hline $2014-2018$ & 1098 & 887 & 158 & 44 & 202 & 9 & Valid \\
\hline Total & 3676 & 2755 & 868 & 44 & 912 & 9 & \\
\hline
\end{tabular}

petitions of babies born in the system's first year (2009), the reviews were completed in early 2015, while petitions of patients born in 2010 or later were processed within the appropriate 5-year windows as described previously. There were 419 eligible cases among those born in 2009, followed by 382 in 2010, 355 in 2011, 361 in 2012, and 351 in 2013, which seems to demonstrate a downward trend (Table 33.1). As of June 2019, 868 (24\%) of the petitions had been deemed ineligible. The reasons for rejection were studied in depth by the Steering Committee of the JOCS-CP. The most common reasons were that the petition did not meet the case-by-case review criteria in that the $\mathrm{pH}$ of umbilical arterial blood exceeded 7.1 and/or the fetal heart rate pattern did not meet the criteria for hypoxia during labor and delivery as specified by the standard compensation agreement. The next most common reasons for rejection were that the cerebral palsy was clearly congenital (i.e., caused by major brain anomalies, chromosomal abnormalities, genetic abnormalities, etc.) and/or caused by conditions that emerged during or after the neonatal period, such as meningitis. Petitions rejected by the Review Committee may petition the Appeal Review Committee by providing a rationale for the initial claim. Of the 181 cases rejected by the Review Committee that appealed, only four petitions had been approved. Thus, the Appeal Review Committee has mostly supported the Review Committee's decisions.

\subsection{Investigation: Identifying Error During Delivery}

Investigation is a primary pillar of the JOCS-CP process aimed not only at preventing cerebral palsy, but also at mitigating conflict and reaching speedy resolutions and settlements (Fig. 33.3). To achieve these goals, an Investigative Report is provided to the relevant childbirth facility and to the patients' guardians/family. Investigative Reports result from intensive scientific investigations using sufficient data from childbirth facilities and input from the patients' guardians/ families. During the introductory and early years of the JOCS-CP, some obstetricians on the frontline who were not engaged in the operation of the JOCS-CP rigorously opposed sharing Investigative Reports with childbirth facilities and families because they feared that the report could provoke conflict and potentially lawsuits, which had not been the case before the JOCS-CP was commenced. On the contrary, another group of obstetricians and patient representatives claimed that the Investigative Reports would meet the families' expectations to learn the cause of cerebral palsy because scientific experts had generated them. Years later, the number of lawsuits did indeed decrease rapidly, as had predicted the latter group of obstetricians and patient representatives.

The JQ has equipped itself with seven subcommittees as working groups to produce the Investigative Reports. Each sub-committee comprises five obstetricians (the chair and four drafting members), two neonatologists, one midwife, 


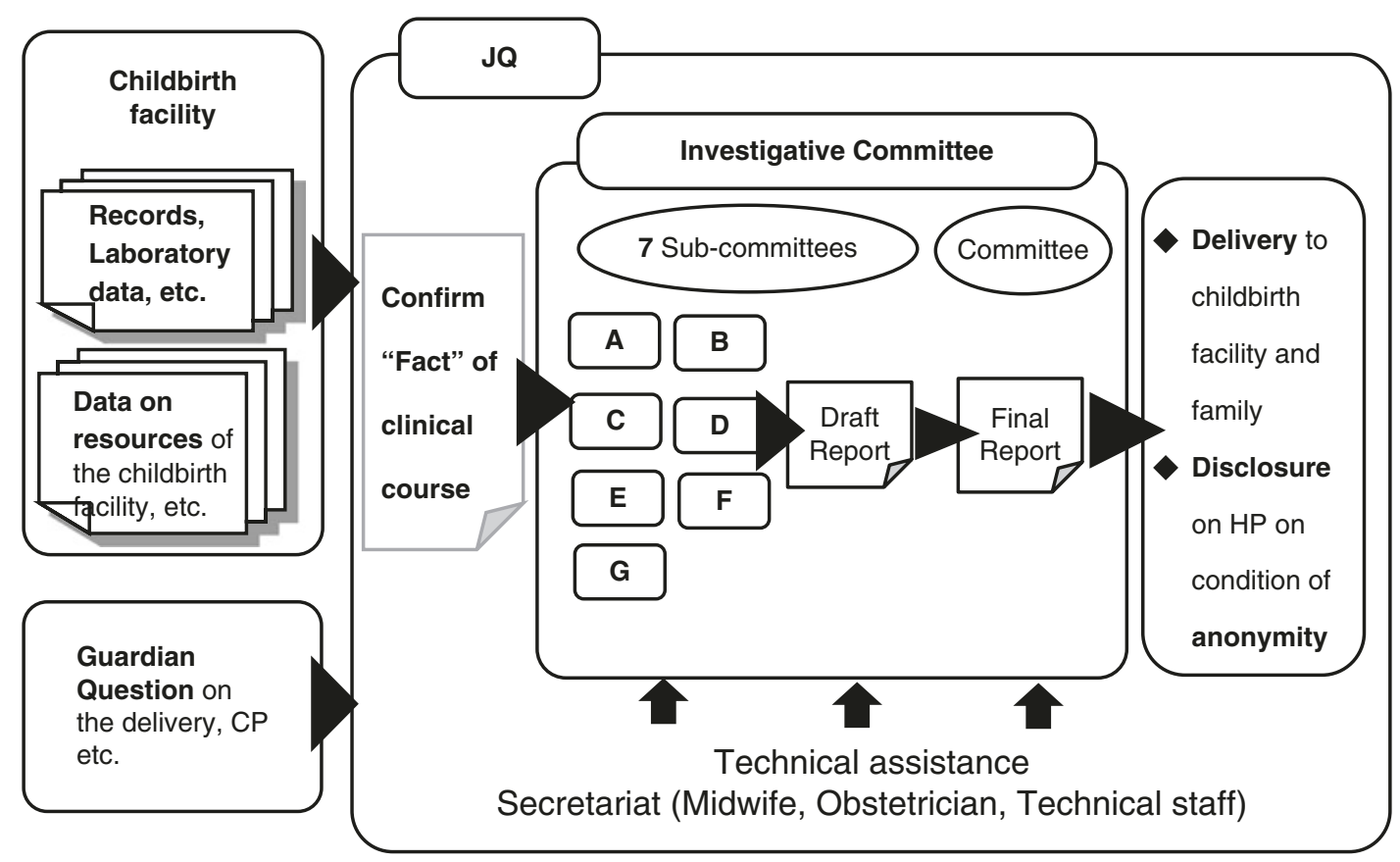

Fig. 33.3 Production of standardized investigative report

and two lawyers (one representing the physician and the other representing the family). The lawyers are appointed from a list of recommendations provided by the Japan Federation of Bar Associations in response to JQ's request. The investigative procedure proceeds as follows. Petitions approved by the Review Committee are sent to the sub-committees, which then draft Investigative Reports. The Investigation Committee handles about 400 such reports per year. The five physicians and the midwife mostly provide medical perspective and the two lawyers draft standardized Investigative Reports that include clear points of contention which are easy for lay people to understand. The reports are structured with tables of contents and uniform language within and across reports to clearly convey meaning. A manual was written to guide the standardization of the investigative work and reporting. The sub-committees produce drafts that are reviewed by the Investigative Committee to finalize them. Summaries of the final Investigative Reports with all personal identifiers deleted are posted on the JOCS-CP website for scientific use and to ensure transparency. As of June 2019, 2369 Investigative Reports had been published. The website includes a keyword search engine so that users can locate cases of interest. Copies of the originals are also available on request with personal identifiers deleted after confirmation by JQ's ethics committee of the reason for the request and so on. Because Japan's Ethical Guidelines for Medical and Health Research Involving Human Subjects, revised on April 1, 2015, might influence the disclosure of Investigative Reports, JQ revised the disclosure process to conform to the revised ethical rules. Since November of 2015, JQ's ethics committee has put into effect revised procedures for disclosure. The concept and policy of producing Investigative Reports focuses on production of an Investigative Report that fully satisfies guardians/ families. Therefore, the goals, concepts, and policies are stated in the manual of investigative work as described below:

1. The Investigative Report does not aim to accuse any party, but to elucidate the probable 
causes of profound cerebral palsy and to specify ameliorative measures in order to prevent similar future events.

2. The Investigative Report should be simple enough for guardians/families, Japanese citizens, and lawyers to understand, and they should be trustworthy.

3. The investigations should be conducted using data gathered during the course of delivery as well as data providing context, such as medical histories of diseases and terms of pregnancy.

4. Delivery procedures, pregnancy management, and so on should be reviewed from a prospective viewpoint in terms of appropriate procedures or management when the event in question, such as hypoxia, occurred.

5. Ameliorative measures for the improvement of perinatal care should be explored from a retrospective viewpoint using all of the data collected from the childbirth facility.

6. The Investigative Committee highly prioritizes its consensus that the prospective viewpoint and the retrospective viewpoint should not be confused to avoid hindsight bias.

\subsection{Controversy on Disclosing Preventability in Individual Cases}

Since the inception of the system, there has been controversy regarding whether to include the "possibility of avoiding cerebral palsy" as determined by the Investigation Committee in an Investigative Report. Obstetricians have worried that stating that cerebral palsy might have been prevented could create conflict, potentially involving lawsuits. Obstetricians were afraid such statements might appear to be an accusation of negligence, despite the systems' goals and its policy of not placing blame. The chair of the Investigative Committee stressed that these statements should not be included in the Investigative Reports because the "possibility of avoidance" is often interpreted as the type of negligence feared by many physicians.
On the other hand, some of the Investigative Committee members, including patient representatives, argued that Investigative Reports should explore the possibilities for avoiding cerebral palsy to help in devising effective preventive measures. Ultimately, these members capitulated and agreed with the chair's position because investigations cannot be carried out without the cooperation and commitment of the medical professionals. The Investigative Committee reached an agreement on December 15, 2009, in which the members in support of the doctors' position presented ways to work on the "possibility of avoidance" issue in the Investigative Report. After controversial arguments, the chair put forth a conciliatory document as a compromise on the issue. The text of that document, which might be a useful reference for investigative work in general, reads as follows.

\subsubsection{Guidance for "The Items to Consider for Better Obstetrical/Perinatal Care" Section of the Investigative Report}

- Preventive measures that might be helpful should be described under "The items to consider for better obstetrical/perinatal care" in the Investigative Report after considering every possible preventive act through intense investigation of procedures and/or management during pregnancy and delivery in comparison with methods of prevention that have been successful in similar cases.

- Items under "The items to consider for better obstetrical/perinatal care" in the Investigative Report should be described using the tone and phrasing exemplified by "It is desirable to be educated and trained in neonatal resuscitation" and "It is strongly recommended that cesarean section or forceps delivery is immediately performed when vacuum delivery is not effective."

- If multiple preventive measures are proposed, they should be presented in order of importance. 
- When cerebral palsy is caused by low-quality or dangerous managerial or procedural practices, the childbirth facility should be urged to improve those practices as soon as possible. To this end, the Investigative Report should clearly state the causes and the relevant preventive measures.

\subsubsection{Guidance for Handling Questions from Guardians/ Families During the Investigative Process}

- In the Investigative Report, the Investigative Committee should answer all questions from the guardian/family from a medical perspective to the greatest extent possible.

- When a guardian/family member asks, "How could the cerebral palsy in our case have been prevented?" the Investigative Committee should provide as complete an answer as possible.

- Answers to questions from the guardian/family are written in a document entitled "Annex."

- The "Annex" must be provided to the childbirth facility and to the guardian/family.

- The contents of an "Annex" should not be disclosed by the JOCS-CP, and it is understood that disclosure by the guardian/family cannot be controlled.

\subsection{Survey on the Investigative Report}

The JOCS-CP initiated the investigative procedures in line with the LDP Framework on the premise that the Investigative Reports would be accepted by the childbirth facilities and the guardians/families and that they would be effective for improving perinatal quality of care and safety. In 2015, a survey on the effectiveness of the Investigative Report was conducted to ascertain whether the Investigative Reports had been embraced by both childbirth facilities and guardians/families. The results found that childbirth facilities and guardians/families mostly supported the Investigative Report's policy, with about $73 \%$ of the facilities and $65 \%$ of the guardians/families responding "very good" or "mostly good." The most frequently cited reason for approving of the Investigative Report was that "analysis was conducted by a third party," suggesting that they attached significant importance to the neutrality and fairness of a third party. Also of note is that, in response to the question about how well the report worked, about $14 \%$ of the guardians/families but just 3\% of the childbirth facilities answered "not very good." The difference in the figures is most likely closely related to the fact that $84 \%$ of guardians/families who answered "not very good" marked "the cause of cerebral palsy was not eventually determined" as the reason, compared to $58 \%$ of the childbirth facilities. Approximately $36.3 \%$ of the Investigative Reports concluded that the cause of cerebral palsy was uncertain or unknown. It seems that the guardian/family respondents tended to expect that the Investigative Committee can always identify probable causes of cerebral palsy given that it is made up of medical experts specializing in perinatal care.

\subsection{Most Frequent Errors}

\section{Prevention-Identification of probable cause of cerebral palsy}

All Investigative Reports are aggregated and put through systematic analysis by the Prevention Committee to create knowledge for the prevention of cerebral palsy through improvements to obstetrical and perinatal care (Fig. 33.4). The JOCS-CP publishes an annual Prevention Report, the latest including 2113 Investigative Reports in its analysis. The JOCS-CP disseminates the preventive measures described in the Prevention Reports as effectively as possible by employing distribution methods which successfully worked in other JQ projects such as Adverse Event Reporting and Learning Systems. The annual Prevention Report specifically includes quantitative and epidemiological analyses of relevant data-such as maternal data, clinical course of pregnancy, delivery and neonatal periods, institutional human resources, and the healthcare provision system-and thematic analysis using data on the causes of cerebral palsy described in the Investigative 


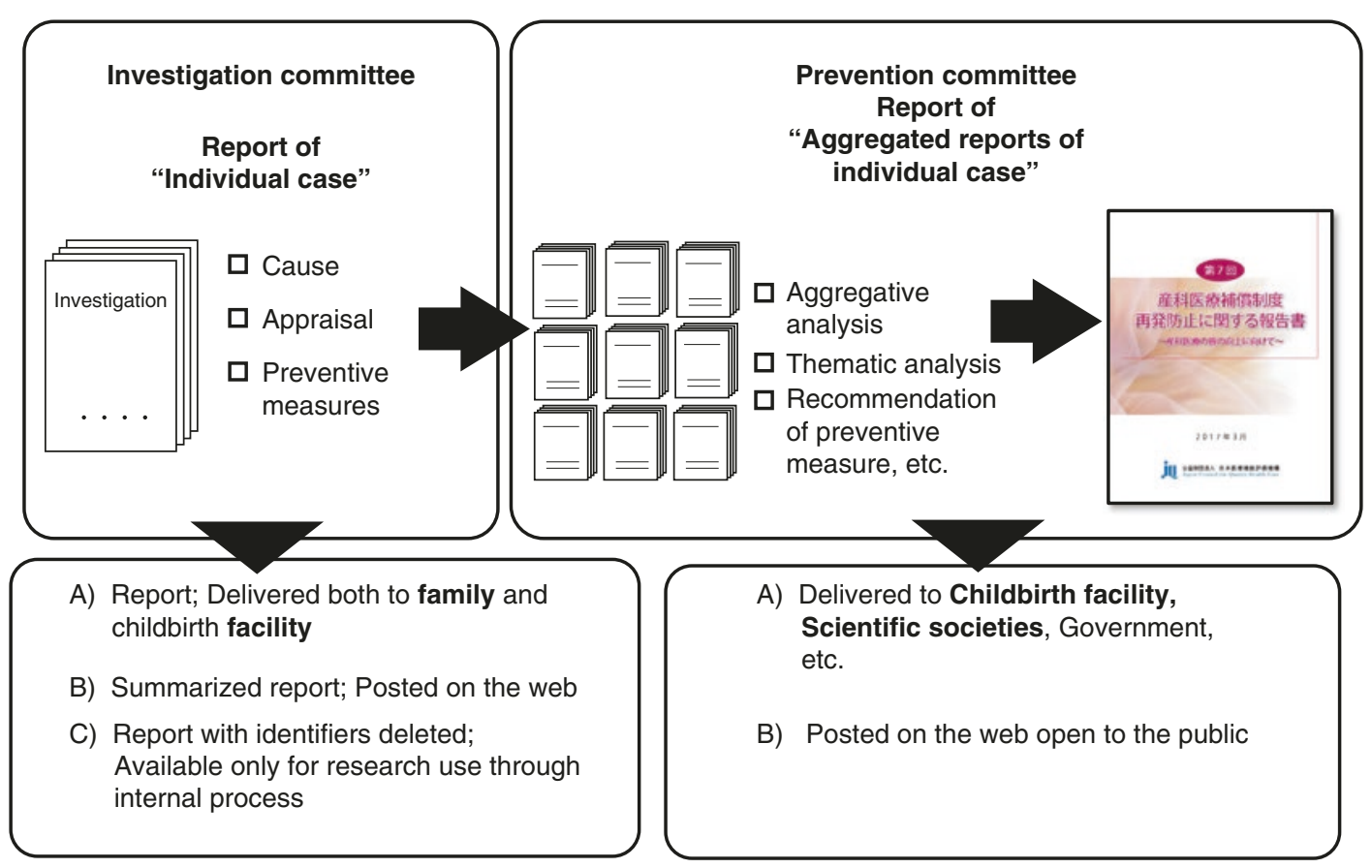

Fig. 33.4 Distributing "Prevention Report" to encourage quality improvement

Reports, fetal heart rate monitoring records, records on the administration of uterinecontracting agents and clinical procedures (such as forced delivery and neonatal resuscitation), availability of medical devices, organizational structure, and other childbirth-related resources and equipment. Aggregate analyses have been used to create tables of probable causes of cerebral palsy approved by the JOCS-CP. The JOCS-CP is the only institution in Japan that has data on probable causes of cerebral palsy on a large scale, although similar results on far smaller scales have been reported. Placental abruption and bleeding, and umbilical factors are the most common causes of cerebral palsy, followed by intrauterine infections and uterine ruptures. However, it should be noted that Investigative Reports failed to determine the causes of the cerebral palsy in $36.3 \%$ of the approved individual cases, suggesting that a significant proportion of cerebral palsy cases have unknown origins despite the current, advanced state of relevant research. The guardians/families and other non-experts generally do not know that knowledge of medical science is limited at this point which potentially increases the likelihood of conflicts between guardians/families and medical professionals. Therefore, it is important for the JOCS-CP to disseminate among our society what is known and what is not known about the causes of cerebral palsy, including the fact that the causes of cerebral palsy have not yet been fully elucidated.

\section{Prevention-Frequent errors and thematic} analysis

The thematic analyses included in the Prevention Report are conducted in accordance with JQ's other projects, such as the Nationwide Adverse Event Reporting and Learning Systems, and are recognized as effective tools for medical professionals to apply to in-depth data on adverse events, in this case cerebral palsy and preventive measures. Twenty themes have been addressed to date. The themes treat error-prone procedures and management which deserve improvement. The themes are as follows:
(a) Vacuum delivery
(b) Uterine contracting agent 
(c) Kristeller maneuver and Pressing maneuver on uterine fundus

(d) Fetal heart rate monitoring during delivery

(e) Umbilical factor except for umbilical prolapse

(f) Umbilical prolapse

(g) Intrauterine infection

(h) Uterine rupture

(i) Placental abruption

(j) Maternal education for early detection of placental abruption

(k) Premature birth

(l) Multiple pregnancy

(m) Pregnancy induced hypertension

(n) Detonated all transfusion syndrome

(o) Neonatal care

(p) Neonatal resuscitation

(q) Case in which neonatal resuscitation is not needed within 5 min after birth

(r) Follow-up statistics of the past themes

(s) Medical chart recording

(t) Emergent transfer system of the pregnant woman and/or baby

Prevention reports are based on thorough study of aggregated Investigative Reports and include assessments of procedures implemented during maternal screening, labor and delivery, and recommended preventive measures. Those elements are presented according to the standardized "Table of contents" in each Investigative Report. The standardization of individual reports enables quantitative and qualitative analysis and a better understanding of the prevention of profound cerebral palsy. The structure of the thematic analyses in the Prevention Report is also carefully standardized.

\subsection{Safety Practices and Implementation Strategy}

The information gleaned from the Prevention Report strongly influences obstetricians and midwives because it is generated from data on cases of cerebral palsy which actually took place in Japanese childbirth facilities. The Prevention
Reports published to date are available on the JOCS-CP website in PDF and CSV file formats. The thematic analysis is displayed in a particularly user-friendly fashion through a list of individual PDFs grouped by theme. In 2017, the Japan Society of Obstetricians and Gynecologists (JSOG) and JAOG revised the guidelines for obstetrical care, entitled "Guidelines for obstetrical practice in Japan." The guidelines address five clinical questions using knowledge gained from thematic analyses of the Prevention Reports and the book on Cardiotocogram interpretation for profound cerebral palsy. Therefore, it is increasingly apparent that the JOCS-CP is a provider of up-to-date knowledge on perinatal care for the medical professionals of Japan's scientific community. The information generated by the Prevention Reports is shared with pregnant women as well as medical professionals for better understanding their pregnancies. One of the leaflets for experts stresses the importance of consent in cases of induced labor using uterinecontracting agents. It recommends that the consent document includes indications of the agents, drug-related side effects, possible treatments related to side effects, alternative drug options, and procedures to ensure patient safety during labor and delivery. Similarly, a leaflet for pregnant women depicts the standard practice of early skin-to-skin contact and highlights key items such as informed, written consent, the safe positioning of the baby, the baby's expected body temperature, and so on. Patient representatives of the Prevention Committee have made enormous contributions to the leaflets for pregnant women, mothers with neonates, and family members.

\subsection{Two Clinical Cases}

The Prevention Report includes not only quantitative analysis but also qualitative and thematic analysis. The latter describes specific facts, procedures, management, and probable cause of cerebral palsy, along with recommendations. Here, we present two cases from the report which are closely related to patient safety. 


\subsubsection{Case 1}

\subsubsection{Clinical Course}

The patient presented with genital bleeding and abdominal stiffness with lower abdominal pain and was admitted to the clinic. She was diagnosed with imminent birth, and ritodrine hydrochloride was therefore administered intravenously. A labor-monitoring device was applied to the patient and dosage of ritodrine hydrochloride was increased in relation to the continuation of symptoms. The baseline fetal heart rate was $130 \mathrm{bpm}$ with late deceleration. On observing the fetal heart rate pattern, the physician paused labor monitoring. However, the monitoring resumed approximately $1 \mathrm{~h}$ and $30 \mathrm{~min}$ later and the device showed that the baseline variability of fetal heart rate (FHR) had decreased and mild variable deceleration manifested. Therefore, the patient was transferred to another hospital, where premature abruption of placenta was suspected. Cardiotocography (CTG) demonstrated a highly decreased FHR and mild variable deceleration. Ultrasonography revealed further thickened placenta. The physician suspected that a cesarean section was emergently needed and therefore suggested and explained the procedure to obtain consent of the patient and a member of their family. Testing for the surgery was conducted in a hurried manner. Subsequently, an emergent cesarean section was initiated approximately $1 \mathrm{~h}$ after consent was obtained. The baby was born 21 min after the cesarean section was initiated, with birth weight of $2540 \mathrm{~g}$, umbilical cord arterial blood $\mathrm{pH}$ of 6.7 , and $\mathrm{BE}$ of $-27 \mathrm{mmol} / \mathrm{L}$. Apgar score was determined to be 1 after $1 \mathrm{~min}$ and 1 after $5 \mathrm{~min}$. Neonatal resuscitation was conducted by Bag-Valve-Mask (BVM) ventilation, tracheal intubation, and chest compression. The baby was diagnosed with premature birth and severe neonatal asphyxia. Pathological testing of the placenta displayed chorioamnionitis. Twelve days after the birth, MRI imaging revealed severe hypoxic encephalopathy (HIE).

\subsubsection{Probable Cause of Cerebral Palsy}

(a) Cerebral palsy was likely caused through hypoxia and academia of the fetus brought on by premature abruption of the placenta.

(b) Intrauterine infection might have affected the placental abruption.

(c) The abruption supposedly took place before admission although it is difficult to identify the time of occurrence precisely.

\subsubsection{Evaluation of Procedures}

(a) Ritodrine hydrochloride was increased and the patient was kept under observation with the clinical findings that fetal heart monitoring revealed decreased variability of the baseline and frequent mild and severe late deceleration. These findings are not commonly seen in clinical practice. The pattern should be interpreted as fetal insufficiency which requires subsequent decision-making by the physician, including cesarean section according to the clinical guidelines.

(b) It is common practice that the clinic would transfer the patient to the hospital based on findings of decreased variability of the baseline and mild variable deceleration.

(c) It is common that, based on the ultrasonography findings, the physician would conduct blood tests and recommend cesarean section, obtaining the written consent of the patient and the family member.

(d) It is a common finding that the fetal heart rate pattern shows decreased variability of the baseline and fluctuates repeatedly by a decrease of $15 \mathrm{bpm}$ for 1 min followed by recovery to normal.

(e) However, it does not reflect common practice that the physician did not plan the cesarean section faster, making the decision to move the patient to the operating theater about $1 \mathrm{~h}$ after obtaining consent.

(f) It is common practice that the physician would plan grade A cesarean section after having identified fetal bradycardia from 
$115 \mathrm{bpm}$ to $80 \mathrm{bpm}$ and complete delivery by cesarean section in $21 \mathrm{~min}$.

\subsubsection{Recommendations}

For the clinic:

(a) Participation in internal and/or external education and training with cardiotocograms is encouraged for better interpretation. In addition, staff may participate in conferences organized by the hospital about the transferred case.

(b) Staff are encouraged to study clinical guidelines on delivery to make correct differential diagnosis of placental abruption from imminent birth.

For the hospital:

(a) Participation in internal and/or external education and training with cardiotocograms is encouraged for better interpretation.

(b) Staff is encouraged to follow clinical guidelines when placental abruption is suspected in a patient.

\subsubsection{Case 2}

\subsubsection{Clinical Course}

The patient was in the clinical course of her first pregnancy and undergoing regular check-ups by the hospital. She was pregnant with dichorionicdiamniotic (DD) twins. The second twin eventually developed cerebral palsy. The patient was obese with a BMI of 33.9 before she became pregnant. She had been diagnosed with diabetes mellitus which was treated with insulin during the previous 5-6 years. She was diagnosed with imminent birth in the 33rd gestational week and treated with ritodrine hydrochloride. The intravenous injections were administered until the 35 th gestational week. In the 36th week, the patient manifested spontaneous membrane rupture and labor pain was noted. The labor was induced by oxytocin as it was too weak to keep in observation. The first twin was delivered after $10 \mathrm{~h}$ and 53 min through a joint maneuver of uterus com- pression and four-round vacuum delivery. Around the time of the first twin's birth, the fetal heart rate pattern of the second twin exhibited decreased variability of the baseline. The findings further deteriorated after the birth of the first twin, showing repetition of late deceleration and bradycardia. The second twin was eventually delivered through four rounds of uterus compressions and six rounds of vacuum delivery. The second twin was born in the 36th gestational week with birth weight of $2610 \mathrm{~g}$. Analysis of umbilical arterial blood sample exhibited a $\mathrm{pH}$ of 6.6 and $\mathrm{BE}-34 \mathrm{mmol} / \mathrm{L}$. The baby's Apgar score was determined to be 0 after 1 min and 0 after $5 \mathrm{~min}$. The baby went into cardiac arrest at birth, and therefore cardiac resuscitation was immediately attempted through manual ventilation, chest compression, tracheal intubation, and injection of adrenaline. Spontaneous heartbeats were observed 14 min after the initiation of the resuscitation. The baby was subsequently moved to a NICU where mechanical ventilation was applied. Hypothermia was initiated for the baby $4 \mathrm{~h}$ after birth; however, it was terminated on Day 1 after birth when ultrasonography of the brain revealed extensive bleeding in the bilateral hypothalamus. As hemoglobin levels dropped to $7.4 \mathrm{~g} / \mathrm{dL}$, blood transfusion was conducted. Brain CT imaging reported (1) high density of the right ventricular wall suggesting subependymal hemorrhage, (2) enlarged ventricules and subaponeurotic hemorrhage, and (3) low density of cerebral white matter bilaterally suggesting previous hypoxia and immaturity of the brain structure.

\subsubsection{Probable Cause of Cerebral Palsy}

(a) The baby developed cerebral palsy probably due to hypoxic conditions and associated fetal circulatory failure which took place during the delivery of the first twin and continued for approximately 40 min eventually causing severe hypoxia in the second baby.

(b) Fetal hypoxia was supposedly caused by rapid uterine contraction after the birth of the first twin followed by increased intrauterine pressure and umbilical factors such as umbilical cord compression. 
(c) Vacuum delivery of the first twin jointly performed with uterine compression maneuver may have caused hypoxia in the second twin.

(d) Furthermore, it cannot be denied that the application of the uterine compression maneuver in the delivery of the second twin contributed as a deteriorating factor.

(e) In addition, it is probable that the loss of circulatory volume due to subaponeurotic hemorrhage and to the cardiac arrest at birth that lasted for $14 \mathrm{~min}$ gave rise to cerebral palsy.

\subsubsection{Evaluation of Procedures}

(a) Management of imminent birth, procedural selection of delivery, description of risk in consent procedure of second baby during delivery of twins, the procedures that were applied when the patient underwent spontaneous rupture of the membrane in the 36th gestational week are all common practice.

(b) In contrast, it does not reflect common practice that the medical staff did not ensure intravenous line until full dilation in the patient with gestational diabetes mellitus who suffered hypoglycemia.

(c) Dosage of oxytocin to begin injection and dosage acceleration strayed from the guidelines.

(d) In terms of judgment and procedure, it is standard that the physician diagnosed the patient with prolonged active phase of delivery and decided to implement vacuum delivery; however, it is not standard that delivery ultimately ended with a contraction period of $43 \mathrm{~min}$ and that the uterine compression maneuver was applied to the delivery of the first twin.

(e) It does not reflect common practice that the physician increased the dosage of oxytocin and did not provide the patient with oxygen amid suspicion of fetal insufficiency.

(f) It is acceptable to select vacuum delivery as the method of emergent delivery for the second twin.

(g) It is controversial from a medical point of view to apply the uterine compression maneuver for the second twin because while some claim that it effectively helps vacuum delivery, others claim that it may prompt uterine rupture or failure of placental circulation.

(h) It is common for the delivery to be conducted in the presence of a pediatrician upon request.

(i) The procedure of neonatal resuscitation was in compliance with standards.

\subsubsection{Recommendations}

For the hospital:

(a) Management of delivery of twins

- It is known that the proceedings of the clinical course of delivery vary even when carefully planned. Therefore, it is desirable that the decision of whether to continue to attempt vaginal delivery or to resort to emergent cesarean section should be made deliberately and swiftly.

- In addition, when attempting vaginal delivery of the second twin, cesarean section should always be a ready option for securing the well-being of the second twin.

- Uterine compression maneuver should be carefully applied during the birth of the first twin, taking into consideration that it may affect placental circulation for the second twin.

(b) Administration of uterine contracting agent

- Prescription and administration of uterine contracting agents should firmly abide by the latest clinical guidelines generated by academic and professional societies.

(c) Pathological testing of placenta

- It is recommended that pathological testing of placenta should be performed as it is vital to explore the cause of neurological symptoms of a baby that may be observed at and after birth.

(d) Management and monitoring system for vaginal delivery of twins

- The physician explained to the patient soon after birth that they had tried vacuum delivery as it would have taken $30 \mathrm{~min}$ to conduct a cesarean section no matter how fast they worked on delivery during the procedure. 
- It is desirable that systems for an emergent cesarean section are always ready as a cesarean section for the second twin is seen with some frequency.

- A notice from the Ministry of Health, Labour and Welfare regarding the improvement of regional capabilities to provide perinatal care states that it is desirable for a regional center for perinatal care to be staffed with obstetricians, anesthesiologists, and other relevant staff to be able to conduct emergent cesarean section within $30 \mathrm{~min}$.

- If this is not possible within the context of the hospital, the hospital should consider whether to conduct delivery in an operating room or conduct the cesarean section in the delivery ward.

For academic and professional society:

(a) It is desirable to produce in-depth guidance for the vaginal delivery of twins.

\subsection{Recommendations}

JOCS-CP has conducted preventive activities, particularly thematic analysis, which produce recommendations related to the aforementioned themes. It is vital for medical professionals to take into account the recommendations for providing high standards of healthcare in the perinatal system. Excerpts from the recommendations are listed below.

\subsubsection{Vacuum Delivery}

For obstetricians:

(a) Vacuum delivery should be done in a timely manner with the appropriate procedures.

- It should be conducted by a trained physician or a physician under guidance of trained physician.

- The physician to conduct vacuum delivery should consider the clinical course, including the mother's well-being, the baby's station, and fetal head rotation, and strictly follow the rules of indication and relevant conditions for implementation.

(b) Procedures of delivery should be reviewed where necessary during delivery.

- Clinical guideline on delivery states that alternative measure such as cesarean section for delivery should be explored in expedited manner when delivery do not proceed with baby's head at zero station. If that is the case, it should be noted that trained staffs are ready for neonatal resuscitation.

(c) It should be recognized that delivery with uterine compression maneuver could bring harm to the well-being of the fetus.

- Uterine compression maneuver should be applied only a couple of times when needed to complete delivery.

(d) A baby delivered through vacuum delivery should be carefully observed for a certain period after birth.

For academic and professional societies:

(a) Education on vacuum delivery should be provided at the institutional level.

(b) Clinical guidelines developed by academic and professional societies should be shared among member physicians.

(c) The guideline should include with more detailed description items to be carefully noted about vacuum delivery and the observation of a baby delivered through vacuum delivery.

\subsubsection{Administration of Uterine Contracting Agents}

For obstetricians:

(a) Indications, condition, and contraindications should be carefully considered. Patient consent should be documented. In case of emergent administration after verbal consent, it should be recorded in writing. 
- Fetal well-being should be reviewed before administration.

- The fetus should be carefully monitored with a fetal heart-monitoring device after the administration of uterine contracting agents because they may cause hypercontraction of the uterus. The administration should be reviewed upon observing bradycardia of the fetus.

- Uterine contracting agents should be prescribed and administered in accordance with the dosage and administration guidelines specified in the package insert.

(b) Concomitant administration of multiple uterine contracting agents.

- PGF2 $\alpha$ and oxytocin should not be concomitantly administered with PGE2. A 1-h interval should be kept between their administrations.

(c) Labor induction procedure amid administration of uterine contracting agents.

- Cervical dilation should precede the administration of uterine contracting agents. Cervical dilating agents and uterine contracting agents should not be concomitantly given to patient.

- A balloon dilator may be applied preceding uterine contracting agents with a 1-h interval.

For academic and professional societies:

(a) Clinical guidelines should detail the dosages of uterine contracting agents and uterine dilating agents and the appropriate intervals of administration.

(b) If possible, a standard should be created for the monitoring of labor with administration of uterine contracting agents.

\subsubsection{Fetal Heart Rate Monitoring}

For medical facilities:

(a) Fetal heart rate should be carefully monitored, paying attention to the following items:

- During the latent phase of labor, no abnormal pattern should be initially observed by continuous monitoring with monitoring device for $20 \mathrm{~min}$. The next period of continuous monitoring should be done within $6 \mathrm{~h}$, with intermittent monitoring every 15-90 min during the same phase.

- In case of lack of monitoring devices in the facility (e.g., a midwife facility), the fetal heart rate should be monitored every 15 min during the latent phase of delivery and every 5 min during the active phase. Monitoring should be done for at least $60 \mathrm{~s}$ after uterine contraction to interpret any change in heart rate due to the contraction.

- Physicians and midwives may conduct continuous monitoring throughout the latent period.

(b) The clinical guidelines should include the requirement for $20 \mathrm{~min}$ of continuous monitoring.

(c) The clinical guidelines should include any other requirements for continuous monitoring of fetal heart rate.

(d) The transducers of the monitoring device should be correctly applied to the patient. The site of application and the belt to fix the transducers may be changed and fastened or loosened as needed for proper functioning.

(e) Recording of fetal heart rate should be done with attention to the following items:

- The time of the recording should be standardized.

- The fetal heart rate should be recorded on paper at a speed of $3 \mathrm{~cm} / \mathrm{min}$ for accurate visualization of the heart rate pattern.

- The fetal heart recording should be stored with the medical chart which must be stored for 5 years by regulation.

- Clinical findings related to the monitoring (e.g., fetal heart rate and status of labor) should be appropriately described on the medical chart.

For academic and professional societies:

(a) The clinical guidelines should be revised to improve understanding of the general procedures of monitoring in delivery and the spe- 
cific monitoring procedures (1) after appreciation of labor, (2) with administration of uterine contracting agents, (3) during TOLAC, (4) after full rupture of membrane, and (5) in high-risk pregnancy such as a prolonged active phase of delivery potentially causing fetal insufficiency. In addition, the revised guidelines should be distributed among medical professionals in perinatal care.

(b) Midwives should have access to facility guidelines on procedures of intermittent fetal heart rate monitoring and should understand the necessity of the periodic application of a monitoring device as well as continuous monitoring.

(c) That the fetal heart rate should be recorded on paper at a rate of $3 \mathrm{~cm} / \mathrm{min}$ should be made widely known in academic and professional societies.

\subsubsection{Care for Placental Abruption}

For pregnant mothers:

(a) Patients should consult childbirth facilities as soon as possible if they perceive symptoms relating to placental abruption, rather than endure them. These symptoms include vaginal bleeding, abdominal pain, stiffness of the abdomen, and decrease or lack of fetal movement.

(b) Pregnant women must pay special attention to relevant symptoms if they may carry risk factors related to placental abruption, including pregnancy-induced hypertension syndrome, past history of placental abruption and trauma caused by traffic accident, and age of 35 years or older.

For obstetricians:

(a) Management of pregnancy

- All patients should be informed of early onset symptoms of placental abruption (i.e., vaginal bleeding, abdominal pain, stiffness of the abdomen, decrease of fetal movement) by the time they reach the 30 th gestational week.

- Health education and attentive health check-ups should be provided to patients who bear risk factors (e.g., pregnancyinduced hypertension) with a physician's full awareness of those factors.

(b) Diagnosis of placental abruption

- Fetal heart rate monitoring device should be applied to the patient for a certain duration (20 min or longer) to check the wellbeing of the fetus on admission of all patients and during any consultation in which the patient manifests abnormal signs.

- The possibility of placental abruption should be considered when symptoms suggestive of imminent birth and any abnormality in the fetal heart rate pattern are observed. Subsequently, differential diagnosis should follow in accordance with the clinical guidelines using ultrasonography, blood tests (i.e., CBC), serum chemistry, blood coagulation, and fetal heart rate monitoring.

- Placental abruption should be clinically examined, noting not only typical manifestations (i.e., abdominal pain, stiffness of the abdomen, vaginal bleeding, decrease or lack of fetal movement) but also atypical ones such as lumber pain. Furthermore, symptoms suggestive of initiation of delivery, such as labor onset and the sensation of water breaking, could be observed.

- All medical professionals relevant to childbirth should receive education and training on CTG interpretation, whether in-house or externally.

(c) Care to provide after the diagnosis with placental abruption is made

- The fetus should be delivered as soon as possible, both under careful maternal management of DIC and fetal management of premature birth. When planning the delivery, the type of forced delivery, 
presence of pediatrician, and necessary transfer of mother and/or baby should be taken into consideration among other factors.

- Neonatal resuscitation should be in line with the latest clinical guidelines. In addition, indication of hypothermia should be deliberated when baby is resuscitated.

- The medical chart may be produced after care and must record details such as complaints of the pregnant mother, internal examination, ultrasonography, fetal heart rate monitoring, transfer of the mother, and the performance of a cesarean section.

(d) System to provide emergent care

- Standardized emergency communication flow should be implemented for prompt treatment of the patient, covering the receipt of a call from a patient who complains of symptoms suggestive of placental abruption (i.e., vaginal bleeding, abdominal pain stiffness of the abdomen, decrease of fetal movement) and its prompt correspondence to medical professionals.

- Institutional standards should be created for the prompt treatment of placental abruption as to prompt forced delivery without transfer, request of maternal and/ or neonatal transfer and presence of pediatrician in reference to context of the institution such as staffing for emergent surgery, care to provide the patient including blood transfusion, neonatal resuscitation, other neonatal care including hypothermia, etc.

- Procedures should be established for emergent calls for medical staff and effective communication of the level of emergency. In addition, emergency systems should be routinely improved through simulation training to provide emergent care.

- Childbirth facilities should be ready in advance to provide care for a patient who has been transferred with placental abruption or probable placental abruption. It is desirable for care plans to be deliberated upon the arrival of the patient, evaluating the well-being of the fetus and placental abruption.

For academic and professional societies:

(a) Research on the cause and early diagnosis of placental abruption is encouraged to aggregate individual cases.

(b) Public outreach is encouraged to let it be widely known that

- Placental abruption is an emergent state of disease which threatens the life of the fetus.

- Placental abruption may have profound consequences for the mother.

- Patients should consult a childbirth facility as soon as possible when they suspect they may suffer the disease.

(c) The development of guidelines for the education of pregnant mothers should be encouraged. The guidelines should outline symptoms which suggest placental abruption and actions they must take.

For central and local governments:

(a) Maternal/fetal transfer systems should be reinforced on a regional scale when they are at risk, as should regional centers for perinatal care. In particular, it is recommended to establish transfer systems beyond prefectural jurisdiction.

(b) Projects should be financed to research causes and early diagnosis of placental abruption.

\section{Further Readings}

1. Japan Council for Quality Health Care, Japan Obstetric Compensation System for Cerebral Palsy (JOCS-CP). Available from: www.sanka-hp.jcqhc. or.jp/index.html (in Japanese).

2. Ushiro S, Suzuki H, Ueda S. Japan Obstetric Compensation System for Cerebral Palsy: Strategic system of data aggregation, investigation, amelioration and no-fault compensation. J Obstet Gynaecol 
Res. 2019;45(3):493-513. https://doi.org/10.1111/ jog. 13906.

3. The Japan Obstetric Compensation System for Cerebral Palsy patterns. Cardiotocograms of cerebral palsy cases-interpretations and considerations of FHR. Part 1 of the book: http://www.sanka-hp.jcqhc. or.jp/documents/statistics/pdf/Cardiotocograms_ of_Cerebral_Palsy_Cases_No.1.pdf. Part 2 of the book: http://www.sanka-hp.jcqhc.or.jp/documents/ statistics/pdf/Cardiotocograms_of_Cerebral_
Palsy_Cases_No.2.pdf. Part 3 of the book: http:// www.sanka-hp.jcqhc.or.jp/documents/statistics/pdf/Cardiotocograms_of_Cerebral_Palsy_ Cases_No.3.pdf. Part 4 of the book: http://www. sanka-hp.jcqhc.or.jp/documents/statistics/pdf/ Cardiotocograms_of_Cerebral_Palsy_Cases_ No.4.pdf.

Open Access This chapter is licensed under the terms of the Creative Commons Attribution 4.0 International License (http://creativecommons.org/licenses/by/4.0/), which permits use, sharing, adaptation, distribution and reproduction in any medium or format, as long as you give appropriate credit to the original author(s) and the source, provide a link to the Creative Commons license and indicate if changes were made.

The images or other third party material in this chapter are included in the chapter's Creative Commons license, unless indicated otherwise in a credit line to the material. If material is not included in the chapter's Creative Commons license and your intended use is not permitted by statutory regulation or exceeds the permitted use, you will need to obtain permission directly from the copyright holder. 